12

\title{
Двумерные кулоновские плазмон-экситоны: релаксация возбуждений
}

\author{
(C) В.А. Кособукин \\ Физико-технический институт им. А.Ф. Иофффе РАН, \\ Санкт-Петербург, Россия \\ E-mail: Vladimir.Kosobukin@mail.ioffe.ru
}

Поступила в Редакцию 7 апреля 2021 г.

В окончательной редакции 7 апреля 2021 г.

Принята к публикации 21 апреля 2021 г.

Представлена теория релаксации двумерных нерадиационных (кулоновских) плазмон-экситонов в близко расположенных тонких слоях металла и полупроводника. В рамках классической электродинамики сформулированы уравнения движения для волн поляризации нерадиационных плазмонов и экситонов при наличии их кулоновской связи и внешнего ближнеполевого источника поляризации. В модели связанных гармонических осцилляторов, представленных полями поляризации возбуждений, решена задача о затухании кулоновских плазмонов, экситонов и плазмон-экситонов. Показано, что дисперсионные ветви нормальных плазмон-экситонных мод испытывают антипересечение (отталкиваются) при резонансе между плазмоном и экситоном. С учетом диссипативного затухания возбуждений и энергоообмена между ними исследован процесс релаксации плазмон-экситонов в зависимости от времени. Теория выявляет важные аналогии динамики плазмон-экситонов и других объектов линейной теории колебаний, таких как механические осцилляторы, резонансные электрические контуры и др.

Ключевые слова: плазмоны, экситоны, кулоновская связь, плазмон-экситоны, волны поляризации, процессы релаксации.

DOI: 10.21883/FTT.2021.08.51171.078

\section{1. Введение}

Коллективные электронные возбуждения в конденсированных средах обусловлены дальнодействием кулоновских сил, которое обеспечивает коррелированное движение огромного числа заряженных частиц. С коллективными возбуждениями (колебаниями) электронов связаны волны диэлектрической поляризации, плазмонной в металлах и экситонной в полупроводниках (диэлектриках). В наноструктурах и метаматериалах металл-полупроводник плазмоны и экситоны могут сосуществовать и влиять друг на друга посредством кулоновских сил [1-5]. В низкоразмерных системах эффекты резонансного взаимодействия поляризационных возбуждений проявляются особенно ярко, поскольку при понижении размерности качественно меняются как квантовые состояния электронов, так и характер кулоновских сил [6,7]. По этой причине неизменно растет научный и практический интерес к низкоразмерным коллективным возбуждениям, а в последние годы и к их смешанным модам.

Для электронных возбуждений в твердых телах особое значение имеют смешанные моды резонансной поляризации и электромагнитного поля, известные как плазмонные и экситонные поляритоны. Поляритоны возбуждаются светом, что позволяет изучать радиационные плазмоны и экситоны оптическими методами [6,7]. Однако кроме поляритонных возбуждений чрезвычайно важны и интересны с физической точки зрения их нерадиационные (кулоновские) аналоги, для которых электромагнитное запаздывание оказывается несущественным $[8,9]$. Кулоновские возбуждения могут играть важную роль в безызлучательном переносе электронных возбуждений, в люминесценции и рассеянии света, в оптике ближнего поля и т.п. К тому же, доля нерадиационных мод в плотности электромагнитных состояний существенно больше, чем доля радиационных мод.

Концепция кулоновских плазмон-экситонов сравнительно проста для систем, включающих ансамбли слоистых субволновых частиц полупроводник-металл, дипольные моды которых безусловно оптически активны [2-4,9]. В планарных наноструктурах непосредственное взаимодействие со светом поляризационных возбуждений, даже поляритонных, сильно затруднено из-за наличия строгих законов сохранения энергии и импульса [7]. Во взаимодействии со светом кулоновские возбуждения могут проявиться в случаях, когда законы сохранения нарушены. В работе [10] кратко обсуждались некоторые особенности кулоновских плазмон-экситонов, в частности, применительно к планарным наноструктурам. К этому лишь добавим, что структуры, поддерживающие низкоразмерные плазмон-экситоны, многовариантны из-за разнообразия состава и геометрии их металлической и полупроводниковой компонент. Для большинства работ, посвященных плазмон-экситонам, характерна констатация общих положений, касающихся взаимодействия плазмонов и экситонов, образования связанных мод, расщепления Раби в их спектре и т. п. (например, [2-5,11-13]). При этом технологически обусловленное различие исследуемых структур, как прави- 
ло, привносит в дискуссию детали. Значительно меньше внимания уделялось изучению процессов плазмонэкситонного энергопереноса и релаксации возбуждений (сравни с [14]), хотя эти явления имеют фундаментальное значение [15]. Исследованию релаксации связанных плазмонов и экситонов посвящена настоящая статья.

Цель этой работы - построение теории зависящих от времени процессов релаксации двумерных кулоновских плазмон-экситонов. Рассмотрение относится к модели близко расположенных тонких слоев металла и полупроводника, поддерживающих двумерные плазмоны и экситоны. С учетом бозонного характера возбуждений свойства плазмон-экситонов обсуждаются на основе представлений классической электродинамики, как делается в кристаллооптике [8]. Задача решается на основе уравнений движения гармонических осцилляторов, которые сформулированы для кулоновски связанных волн поляризации двумерных плазмонов и экситонов при наличии вынуждающей силы. Содержание работы заключается в следующем. Модель и постановка задачи представлены в разделе 2, общее решение задачи и анализ спектра даны в разделе 3. В разделе 4 для плазмон-экситонов обсуждаются процессы релаксации, результаты численно анализируются в разделе 5 .

\section{2. Модель и основные уравнения}

В качестве структурной модели для изучения плазмон-экситонов рассматриваем расположенные рядом слои металла и полупроводника, поддерживающие двумерные (2D) плазмоны и экситоны соответственно. Такая модель может относиться к наноструктурам металл-полупроводник с узкими квантовыми ямами и к актуальным структурам с атомарно-тонкими слоями металла и полупроводника. Предметом нашего изучения служат процессы релаксации кулоновских 2D-плазмонэкситонов в зависимости от времени.

Предполагаем, что в тонких слоях слоях металла и полупроводника могут возбуждаться двумерные плазмонная $\mathbf{P}^{(1)}$ и экситонная $\mathbf{P}^{(2)}$ поляризации, которые отмечаются далее верхними индексами 1 и 2 соответственно. Поля поляризации $\mathbf{P}^{(1)}$ и $\mathbf{P}^{(2)}$, имеющие резонансные частоты в оптическом диапазоне, взаимодействуют друг с другом по закону Кулона. Фоновая (при $\mathbf{P}^{(1)}=\mathbf{P}^{(2)}=0$ ) диэлектрическая постоянная среды $\varepsilon_{b}$ однородна и изотропна во всем пространстве. Уравнения колебаний кулоновски связанных гармонических осцилляторов, роль которых играют волны поляризации $\mathbf{P}^{(1)}$ и $\mathbf{P}^{(2)}$, формулируются далее в рамках классической электродинамики.

В плоской геометрии двумерное коллективное возбуждение с волновым вектором $\boldsymbol{\kappa}=\left(\kappa_{x}, \kappa_{y}\right)$ можно представить волной диэлектрической поляризации вида

$$
\mathbf{P}(\mathbf{r}, t)=\overline{\mathbf{P}}(\boldsymbol{\kappa}, \omega) \delta(z) e^{i \kappa \rho-i \omega t}
$$

Такая волна распространяется с вектором $\boldsymbol{\kappa}$ в направлениях трансляционной симметрии с $\boldsymbol{\rho}=(x, y)$

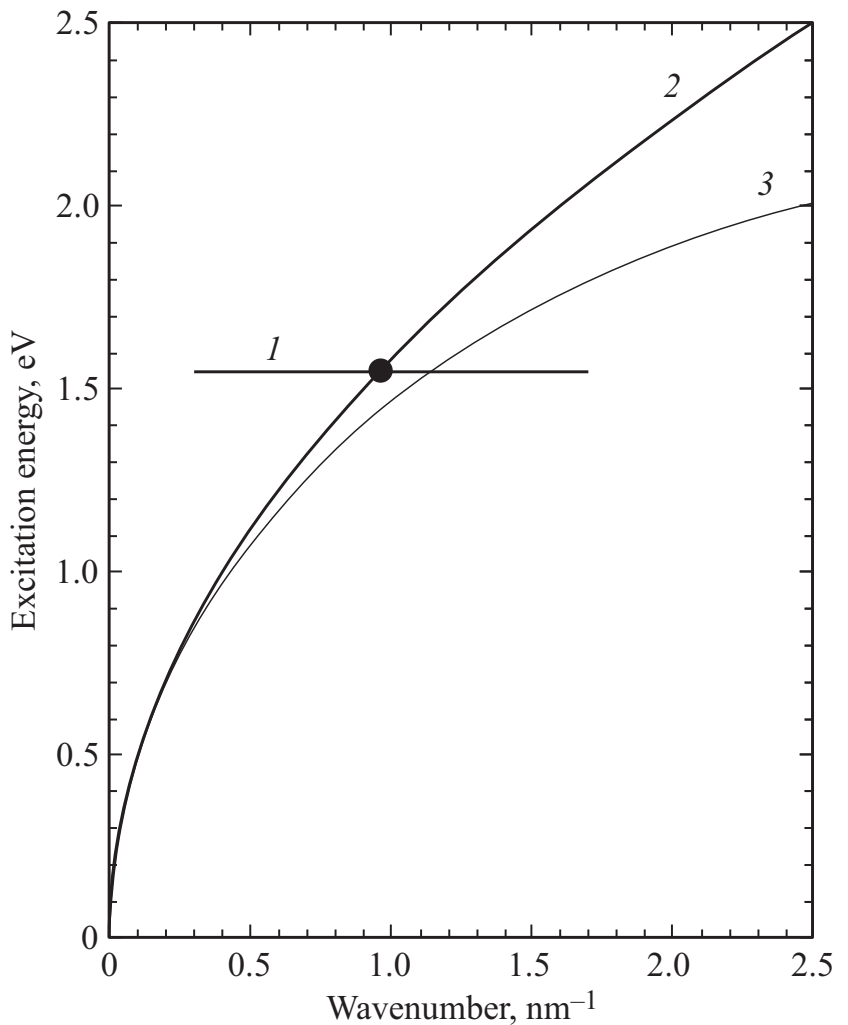

Рис. 1. Законы дисперсии 2D-экситонов $\hbar \omega_{0}(1)$ и плазмонов $\hbar \omega_{2 \mathrm{D}}(2)$ в зависимости от волнового числа $\kappa$. Точка пересечения экситонной и плазмонной дисперсионных ветвей соответствует условию $\omega_{0}=\omega_{2 \mathrm{D}}(\kappa)$ плазмон-экситонного резонанса. Вычислено с параметрами, соответствующими слою металла в среде с $\varepsilon_{b}=12.5$ и слою полупроводника с $\hbar \omega_{0}=1.55 \mathrm{eV}$. Кривая 3 изображает закон дисперсии квазипродольных плазмонов в тонкой $(L=0.7 \mathrm{~nm})$ пленке $\mathrm{Ag}$, находящейся в $\mathrm{GaAs}$, сравни с [10].

и локализована вблизи плоскости $z=0$ в направлении ограничения движения $z$. Для волн вида (1) закон дисперсии предполагается изотропным по направлениям $\boldsymbol{\kappa}$ в плоскости $x y$, при этом для кулоновских (квазистатических) мод $|\boldsymbol{\kappa}| \gg k_{0}=\omega / c$, где $\omega-$ частота, $c-$ скорость света. С поляризацией (1) связана волна плотности электрического заряда $-\rho(\mathbf{r})=\operatorname{div} \mathbf{P}(\mathbf{r}) \sim\left[\bar{P}_{\kappa} i \kappa \delta(z)+\bar{P}_{z} \delta^{\prime}(z)\right] e^{i \kappa \rho}$. Собственное электрическое поле $\mathbf{E}(z, \kappa)$ волны поляризации (1) с $\boldsymbol{\kappa}=\kappa \mathbf{e}_{x}$ имеет компоненты $x$ и $z$, затухающие по закону $E(z, \kappa) \sim e^{-\kappa|z|}$ при увеличении расстояния $|z|$.

В двумерном электронном газе существуют продольные плазмоны с законом дисперсии $\omega_{2 \mathrm{D}}(\kappa) \sim \sqrt{\kappa}[16]$. Дисперсионная ветвь $\omega_{2 \mathrm{D}}(\kappa)$, представленная кривой 1 на рис. 1, показывает, что 2D-плазмоны имеют бесщелевой спектр с нулевой частотой при $\kappa=0$, а их дисперсия определяется кулоновскими силами во всем диапазоне собственных частот. Плазмонная ветвь не пересекается со световыми прямыми $c \kappa / \sqrt{\varepsilon_{b}}>\omega_{2 \mathrm{D}}(\kappa)$, т.е. 2D-плазмоны не возбуждаются светом непосред- 
ственно. Однако двумерные плазмоны можно возбудить с помощью зонда, имеющего в плоскости $x y$ широкий пространственный спектр с $|\boldsymbol{\kappa}| \gg k_{0}$. Это может быть пучок заряженных частиц, в том числе и токовый, ближнеполевой зонд, атомные агрегаты, нанонеоднородности и т. п., но эффективность возбуждения плазмонов или/и экситонов разными способами может сильно различаться. Подчеркнем, что дальнейшее обсуждение относится к плазме металлов, а 2D-плазмоны полупроводниковых квантовых ям, имеющие инфракрасный спектр, из рассмотрения исключаются. В отличие от 2D-плазмонов, квази 2D-экситоны имеют спектр, который определяется частотой оптического перехода $\omega_{0}$ при $\kappa=0$.

Кулоновские экситоны с $\kappa \gg k_{0}$ могут образовать связанные состояния с 2D-плазмонами. Для этого законы дисперсии парциальных мод (плазмона и экситона) должны удовлетворять условию резонанса $\omega_{\text {plasmon }}(\boldsymbol{\kappa})=\omega_{\text {exciton }}(\boldsymbol{\kappa})$, которому на рис. 1 соответствует пересечение дисперсионных ветвей 1 и 2 . В случае нерадиационных парциальных возбуждений смешанные плазмон-экситонные моды тоже являются кулоновскими, и для их возбуждения требуются упомянутые выше источники поля с широким пространственным спектром $\left(\kappa \gg k_{0}\right)$.

Рассмотрим вначале плазмоны в ультратонком слое металла с однородной двумерной плотностью $n_{2 \mathrm{D}}$ электронного газа. В этой плазме для амплитуды волны поляризации $\mathbf{P}^{(1)}$ с вектором $\boldsymbol{\kappa}$ в плоскости $z=0$ локальное уравнение линейного отклика на полное поле $\mathbf{E}$ имеет вид (сравни с [10]):

$$
-\left(\omega^{2}+i \omega \Gamma_{1}\right) \mathbf{P}^{(1)}(z, \boldsymbol{\kappa}, \omega)=\frac{\omega_{p}^{2} L}{4 \pi} \delta(z) \mathbf{E}(z, \boldsymbol{\kappa}, \omega) .
$$

Здесь $\omega_{p}^{2}=4 \pi e^{2} n_{3 \mathrm{D}} / m$ - плазменная частота электронов в металле, $L-$ эффективная толщина слоя плазмы с $n_{2 \mathrm{D}}=n_{3 \mathrm{D}} L$, a $e, m$ и $\Gamma_{1}$ обозначают абсолютный заряд, эффективную массу и обратное время релаксации электронов. Представление отклика вырожденного электронного газа в форме (2) с дельта-функцией в правой части для 2D-плазмонов известно в литературе [16,17]. Соотношение $(2)$ можно получить при $\kappa L \ll 1$ в феноменологической теории отклика квазипродольных поверхностных плазмонов пленки металла толщиной $L$ [10], их закон дисперсии 3 показан на рис. 1. Переход к 2D-плазмонам в пленке при уменьшении толщины $L$, составляющей небольшое число моноатомных слоев, обсуждался также в микроскопической теории [18]. Для кулоновских плазмонов модель должна удовлетворять следующим физическим ограничениям: $k_{0} \ll \kappa \ll k_{F}$, где $k_{F}$ - волновое число Ферми.

Для волны экситонной поляризации с вектором $\boldsymbol{\kappa}$ в тонком слое полупроводника материальное соотношение представим в форме

$$
\left(\omega_{0}^{2}-\omega^{2}-i \omega \Gamma_{2}\right) \mathbf{P}^{(2)}(z, \boldsymbol{\kappa}, \omega)=\Omega^{2} l \delta(z-h) \mathbf{E}(z, \boldsymbol{\kappa}, \omega) .
$$

Здесь $\omega_{0}$ - частота 2D-экситона, $\Gamma_{2}-$ параметр его нерадиационного затухания. Экситонный отклик (3) на полное электрическое поле определяется константой $\Omega^{2}$, a $l$ - характерная толщина экситонного слоя с центральной плоскостью $z=h$, где $h-$ расстояние между плазмонным и экситонным слоями. Такой подход применим для экситонов в квантоворазмерных структурах и в актуальных структурах с ультратонкими слоями металла и полупроводника. Чтобы применить теорию к квази 2D-экситонам Ванье в полупроводниках со структурой $\mathrm{ZnS}$, можно положить $\Omega^{2}=\omega_{0} \omega_{L T} \varepsilon_{b} /(2 \pi)$, где $\omega_{0}-$ частота экситона, а $\omega_{L T}$ выражается через межзонный матричный элемент оператора импульса $[6,10]$.

Согласно (2) и (3) распределение 2D-поляризации парциальных возбуждений обоих типов по нормали к слою формально описывается дельта-функцией: $P^{(n)}(z) \sim \bar{L}_{n} \delta\left(z-z_{n}\right)$, где $\bar{L}_{n}-$ характерная длина локализации возбуждения. Переход к дельта-функции в материальных уравнениях оправдан, если электрическое поле, связанное с поляризацией (2) или (3), рассматривается вне области реальной поляризации. Для квази 2D-экситонов переход к форме (3), аналогичной (2), является результатом отказа [19] от нелокального описания отклика $\mathbf{P}^{(2)}[6,20]$ и замены $\psi(z) \rightarrow \sqrt{l} \delta(z)$ огибающей волновой функции 2D-экситона. Обоснованием такого подхода может служить, в частности, факт, что наблюдаемые оптические величины, например, коэффициент отражения, выражаются через интегралы от дипольной поляризации (от функции $\psi)[6,10]$. С теоретической точки зрения такой интеграл малочувствителен к деталям в распределении $\psi$ поля поляризации, а на практике он входит в качестве константы в некоторый подгоночный параметр, определяемый из данных эксперимента (сравни с теорией [21]).

Сформулируем на основе соотношений (2) и (3) зависящие от времени уравнения движения для полей поляризации. Для этого в соотношениях сделаем замены $(-i \omega)^{s} \rightarrow d^{s} / d t^{s} \quad(s=1,2)$, соответствующие преобразованию Фурье по времени функций $P_{\alpha}^{(n)}(z, \kappa, t)$. Далее учитываем, что поляризация $\mathbf{P}^{(n)}\left(z^{\prime}, \kappa, t\right)$ плазмонов $(n=1)$, экситонов $(n=2)$ или внешнего источника $(n=0)$ порождает в плоскости с координатой $z$ фоновой среды электрическое поле [10]:

$$
E_{\alpha}^{(n)}(z, \kappa, t)=\sum_{\beta} \int d z^{\prime} g_{\alpha \beta}\left(z-z^{\prime}, \kappa\right) P_{\beta}^{(n)}\left(z^{\prime}, \kappa, t\right) .
$$

В формуле (4) $g_{\alpha \beta}\left(z-z^{\prime}, \kappa\right)-$ тензорные компоненты функции Грина в $(z, \kappa)$-представлении, вычисленные при $\mathbf{P}^{(n)}=0$ в квазистатическом приближении (при неучете электромагнитного запаздывания) для однородной фоновой среды с проницаемостью $\varepsilon_{b}[10]$. Полное поле $\mathbf{E}$, стоящее в правых частях соотношений (2) и (3), является суперпозицией полей $\mathbf{E}^{(n)}$ вида (4). 
С учетом формул (4) из уравнения (2) для поляризации плазмонов с волновым вектором $\boldsymbol{\kappa}=\kappa \mathbf{e}_{x}$ находим

$$
\begin{gathered}
\left(\frac{d^{2}}{d t^{2}}+\Gamma_{1} \frac{d}{d t}+\omega_{11}^{2}(\kappa)\right) P_{\alpha}^{(1)}(z, t)=\frac{\omega_{P}^{2} L}{4 \pi} \delta(z) \\
\times \sum_{n=0,2} \sum_{\beta} \int d z^{\prime} g_{\alpha \beta}\left(z-z^{\prime}\right) P_{\beta}^{(n)}\left(z^{\prime}, t\right) .
\end{gathered}
$$

Частота $\omega_{11}$ - это закон дисперсии продольных 2D-плазмонов

$$
\omega_{2 \mathrm{D}}(\kappa)=\left(\frac{2 \pi e^{2} n_{2 \mathrm{D}}}{m \varepsilon_{b}} \kappa\right)^{1 / 2},
$$

полученный при учете по формуле (4) собственного продольного электрического поля плазмонов в слое электронного газа с 2D-плотностью $n_{2 \mathrm{D}}$. Слагаемые с $n=0$ и 2 в правой части (5) выражают электрические поля внешнего источника и экситонов, действующие на плазмон.

Аналогично из соотношения (3) с учетом (4) для экситонной поляризации с $\boldsymbol{\kappa}=\kappa \mathbf{e}_{x}$ находим

$$
\begin{aligned}
\left(\frac{d^{2}}{d t^{2}}\right. & \left.+\Gamma_{2} \frac{d}{d t}+\omega_{22}^{2}(\kappa)\right) P_{\alpha}^{(2)}(z, t)=\Omega^{2} l \delta(z-h) \\
& \times \sum_{n=0,1} \sum_{\beta} \int d z^{\prime} g_{\alpha \beta}\left(z-z^{\prime}\right) P_{\beta}^{(n)}\left(z^{\prime}, t\right) .
\end{aligned}
$$

Здесь частота $\omega_{22} \approx \omega_{0}$ в дополнение к $\omega_{0}$ из (3) учитывает эффект собственного электрического поля (4) экситона [10].

Перенесем в левую часть уравнения (5) члены, связанные с поляризацией $\mathbf{P}^{(2)}$, а в левую часть уравнения (7) - члены, связанные с поляризацией $\mathbf{P}^{(1)}$. При наличии поляризации $\mathbf{P}^{(0)}$ внешнего источника это дает систему двух неоднородных интегральных по $z$ и дифференциальных по $t$ уравнений движения для взаимодействующих мод поляризации 2D кулоновских плазмонов и экситонов.

В качестве иллюстрации внешней поляризации напишем выражение $\mathbf{P}^{(0)}(\mathbf{r}, t)=\boldsymbol{\mu}(t) \delta(\boldsymbol{\rho}) \delta\left(z-z_{0}\right)$ [10], которое соответствует источнику ближнего поля с дипольным моментом $\boldsymbol{\mu}(t)$, расположенным в точке $\mathbf{r}_{0}=\left(0,0, z_{0}\right)$. Чтобы учесть действие диполя на плазмоны и экситоны, следует подставить в правые части уравнений (5) и (7) Фурье-компоненту

$$
\mathbf{P}^{(0)}(z, \kappa, t)=\boldsymbol{\mu}(t) \delta\left(z-z_{0}\right),
$$

которая в случае квазиточечного диполя от $\kappa$ не зависит.

\section{3. Общее решение задачи}

3.1. Уравнения движения. Найдем решение полученной выше системы уравнений с неизвестными $\mathbf{P}^{(1)}$ и $\mathbf{P}^{(2)}$ в левой части и с поляризацией $\mathbf{P}^{(0)}-$ в правой. Для
2D-плазмонов и экситонов рассматриваем продольные волны поляризации $P_{\alpha}^{(n)}(z, \kappa)=\delta_{\alpha x} P_{x}^{(n)}(z, \kappa)$ с $n=1$ и 2 в направлении распространения $\boldsymbol{\kappa}=\kappa \mathbf{e}_{x}$, при этом $P_{z}^{(n)}(z, \kappa)=0$. Преобразуем интегральные по $z$ уравнения (5) и (7), полагая

$$
\begin{gathered}
P_{x}^{(1)}(z, t)=w_{1}(t) L \delta(z), \\
P_{x}^{(2)}(z, t)=w_{2}(t) l \xi \delta(z-h),
\end{gathered}
$$

где $h>0$, а безразмерный множитель $\xi$ определим ниже. В результате получаем систему дифференциальных по $t$ уравнений

$$
\left.\begin{array}{l}
\left(\frac{d^{2}}{d t^{2}}+\Gamma_{1} \frac{d}{d t}+\omega_{11}^{2}\right) w_{1}(t)+\omega_{12}^{2} \xi w_{2}(t)=f_{1}(t), \\
\omega_{21}^{2} w_{1}(t)+\left(\frac{d^{2}}{d t^{2}}+\Gamma_{2} \frac{d}{d t}+\omega_{22}^{2}\right) \xi w_{2}(t)=f_{2}(t)
\end{array}\right\}
$$

для неизвестных амплитуд $w_{1}(t)$ плазмонов и $w_{2}(t)$ экситонов с заданным $\kappa$. В левую часть системы уравнений (10) входят частоты

$$
\left.\begin{array}{l}
\omega_{11}^{2}(\kappa)=\frac{2 \pi e^{2} n_{2 \mathrm{D}}}{m \varepsilon_{b}} \kappa=\omega_{2 \mathrm{D}}^{2}, \omega_{12}^{2}(\kappa)=\omega_{2 \mathrm{D}}^{2} \frac{l}{L} e^{-\kappa h}, \\
\omega_{21}^{2}(\kappa)=\frac{2 \pi}{\varepsilon_{b}} \Omega^{2} \kappa L e^{-\kappa h}, \omega_{22}^{2}(\kappa)=\omega_{0}^{2}+\frac{2 \pi}{\varepsilon_{b}} \Omega^{2} \kappa l .
\end{array}\right\}
$$

Функции $f_{n}(t)$ в правой части уравнений (10) выражают действие внешнего источника поляризации (8) на плазмон и экситон соответственно.

Для решения системы уравнений движения (10) используем операционный метод [22], основанный на преобразовании Лапласа

$$
W_{n}(p)=\int_{0}^{\infty} d t e^{-p t} w_{n}(t),
$$

которое для функции-оригинала $w_{n}(t)$ дает изображение $W_{n}(p)$ с комплексным $p$. Преобразование Лапласа уравнений (10) дает систему алгебраических уравнений

$$
\left.\begin{array}{l}
\left(p^{2}+p \Gamma_{1}+\omega_{11}^{2}(\kappa)\right) W_{1}(p)+\Delta \omega^{2}(\kappa) W_{2}(p)=M_{1}(p)+F_{1}(p), \\
\Delta \omega^{2}(\kappa) W_{1}(p)+\left(p^{2}+p \Gamma_{2}+\omega_{22}^{2}(\kappa)\right) W_{2}(p)=M_{2}(p)+F_{2}(p)
\end{array}\right\}
$$

для изображений $W_{n}(p)$. В правую часть системы (13) входят выражения

$$
M_{n}(p)=\left(p+\Gamma_{n}\right) w_{n}^{0}+\dot{w}_{n}^{0}
$$

которые определяются начальными (при $t=+0$ ) значениями $w_{n}^{0} \equiv w_{n}(0)$ и $\dot{w}_{n}^{0} \equiv \dot{w}_{n}(0)$ функций-оригиналов $w_{n}(t)$ из (9); точкой сверху отмечаются производные по $t$. Функциям-оригиналам $f_{n}(t)$ из (10) для внешней поляризации (8) при $t>0$ в формулах (13) соответствуют изображения $F_{n}(p)$. Если $F_{n}(p)=0$, то 
уравнения (13) определяют вклады $W_{n}(p)$ плазмонов и экситонов в собственные плазмон-экситонные моды, а при $F_{n}(p) \neq 0$ уравнения (13) описывают вынужденные колебания.

Заметим, что уравнения (13) получены в результате перехода к симметрической матрице в переменных $W_{1}$ и $W_{2}$ при использовании в формулах (10) коэффициента $\xi=\omega_{21} / \omega_{12}$, равного $\xi=\left(\Omega / \omega_{2 \mathrm{D}}\right) \sqrt{2 \pi \kappa l / \varepsilon_{b}}(L / l)$. При этом для недиагональных матричных элементов $\Delta \omega^{2}=\omega_{12} \omega_{21}$ в (13) имеем

$$
\Delta \omega^{2}(\kappa)=\omega_{2 \mathrm{D}}(\kappa) \Omega \sqrt{\frac{2 \pi}{\varepsilon_{b}} \kappa l} e^{-\kappa h} .
$$

Величина (15) является немонотонной функцией $\kappa$ с максимумом, положение которого зависит от геометрических параметров модели.

В общем случае решение системы уравнений (13) с $N_{n}=M_{n}+F_{n}$ в правой части имеет вид

$$
\begin{gathered}
W_{1}(p)=\frac{1}{D(p)}\left\{\left(p^{2}+p \Gamma_{2}+\omega_{22}^{2}\right) N_{1}(p)-\Delta \omega^{2} N_{2}(p)\right\}, \\
W_{2}(p)=\frac{1}{D(p)}\left\{-\Delta \omega^{2} N_{1}(p)+\left(p^{2}+p \Gamma_{1}+\omega_{11}^{2}\right) N_{2}(p)\right\} .
\end{gathered}
$$

Знаменатель

$D(p)=\left(p^{2}+p \Gamma_{1}+\omega_{11}^{2}(\kappa)\right) \cdot\left(p^{2}+p \Gamma_{2}+\omega_{22}^{2}(\kappa)\right)-\Delta \omega^{4}$

определяет спектр связанных мод, которые образуются при взаимодействии между плазмонами и экситонами. Это взаимодействие определяется значением константы связи $\Delta \omega$ из (15).

Вклады плазмонов $w_{1}(t)$ и экситонов $w_{2}(t)$ в плазмонэкситоны получаются в результате обратного преобразования Лапласа $W_{n}(p) \rightarrow w_{n}(t)$ изображений (16) и (17) с учетом (18). При обращении преобразования Лапласа учитывается полюсная структура функций $W_{n}(p)$ комплексного переменного $p$. Функции $W_{n}(p)$ из $(16)-(18)$ имеют простые полюса $p_{k}=p_{k}^{\prime}+i p_{k}^{\prime \prime}$ с номерами $k=1-4$, которые являются корнями уравнения $D(p)=0$ для полинома (18). Поскольку коэффициенты полинома $D(p)$ положительны, имеются две пары комплексно сопряженных корней

$$
p_{1}=-g_{\mathrm{I}}-i u_{\mathrm{I}}=p_{2}^{*}, \quad p_{3}=-g_{\mathrm{II}}-i u_{\mathrm{II}}=p_{4}^{*},
$$

которые являются полюсами функции $D^{-1}(p)=$ $=\prod_{k=1}^{4}\left(p-p_{k}\right)^{-1}$, входящей в (16) и (17). Оригиналы функций (16) и (17) при $t>0$ получаются в результате обратного преобразования Лапласа $W_{n}(p) \rightarrow w_{n}(t)$, которое при использовани теории вычетов дает [22]:

$$
w_{n}(t)=2 \operatorname{Re} \sum_{p_{k}} \lim _{p \rightarrow p_{k}} e^{p t}\left(p-p_{k}\right) W_{n}(p) .
$$

Здесь предполагается суммирование по тем полюсам $p_{k}=p_{k}^{\prime}+i p_{k}^{\prime \prime}$ функции $W_{n}(p)$, которые имеют отрицательные мнимые части, а именно по $p_{1}, p_{3}$ из (19). Найденные в результате вычисления $(20)$ функции $w_{n}(t)$ определяют согласно (9) зависящие от времени вклады плазмонной и экситонной поляризации в смешанные плазмон-экситонные моды. Заметим, что при учете возбуждения плазмон-экситонов внешним источником функции $F_{n}(p)$ привносят в (16) и (17) дополнительные к (19) полюса, связанные с частотами колебаний внешней поляризации (8).

3.2. Спектр плазмон-экситонов. В отсутствие внешнего источника поляризации $\left(F_{n}=0\right)$ собственный спектр плазмон-экситонов определяется корнями уравнения $D(-i \omega)=0$ для полинома (18). Найдем из этого уравнения явные выражения для входящих в (19) частот $u_{v}$ и параметров затухания $g_{v}$ смешанных плазмон-экситонных мод, которые нумеруем римскими цифрами $v=$ I, II. Предполагаем, что парциальные плазмоны и экситоны затухают слабо, т. е. $\Gamma_{n} / \omega_{n n} \ll 1$ в (18). В нулевом приближении $\left(\Gamma_{n}=0\right)$ для двух нормальных мод плазмонэкситонов получаем законы дисперсии

$$
u_{\mathrm{I}, \mathrm{II}}^{2}(\kappa)=\frac{\omega_{11}^{2}+\omega_{22}^{2}}{2} \mp \sqrt{\frac{\left(\omega_{11}^{2}-\omega_{22}^{2}\right)^{2}}{4}+\Delta \omega^{4}} .
$$

Затем в низшем приближении по малым $\Gamma_{n}$ находим параметры затухания

$$
g_{v}(\kappa)=\frac{\Gamma_{1}\left(\omega_{22}^{2}-u_{v}^{2}\right)+\Gamma_{2}\left(\omega_{11}^{2}-u_{v}^{2}\right)}{2\left(\omega_{11}^{2}+\omega_{22}^{2}-2 u_{v}^{2}\right)}
$$

для плазмон-экситонов с частотами $u_{v}$. В результате формулах (19) имеем $u_{v}>0$ из (21) и $g_{v}>0$ из (22).

Выражения (21) и (22) приводят к следующим общим выводам о спектре плазмон-экситонов. Вблизи резонанса $\omega_{11}=\omega_{22}\left(\omega_{2 \mathrm{D}}=\omega_{0}\right.$ на рис. 1$)$ поведение частот $u_{\mathrm{I}, \mathrm{II}}(\kappa)$ и параметров затухания $g_{\mathrm{I}, \mathrm{II}}(\kappa)$ определяется константой связи (15). При $\omega_{11}=\omega_{22}$ дисперсионные ветви $u_{\mathrm{I}, \mathrm{II}}(\kappa)$ из (21) в зависимости от $\kappa$ демонстрируют антипересечение (отталкивание) частот $u_{\mathrm{II}}-u_{\mathrm{I}}=\Delta \omega^{2} / \omega_{11}$, при этом $u_{\mathrm{I}}<\omega_{11}, \omega_{22}<u_{\text {II }}[10]$. В области антипересечения параметры затухания (22) плазмон-экситонов с $v=$ I и II сближаются, так что $g_{\mathrm{I}}=g_{\mathrm{II}}=\left(\Gamma_{1}+\Gamma_{2}\right) / 4$ для мод с частотами $u_{\text {I и }} u_{\text {II }}$ при условии $\omega_{11}=\omega_{22}$.

Далее мы рассмотрим зависящие от времени процессы релаксации плазмон-экситонов, обусловленные диссипативным затуханием парциальных возбуждений.

\section{4. Релаксация плазмон-экситонов}

Обсудим теперь применение представленной выше теории к изучению релаксации нерадиационных плазмон-экситонов вследствие затухания плазмонов и экситонов. В уравнениях (16) и (17) полагаем $F_{n}=0$, тогда величины $N_{n}=M_{n}$ определяются начальными амплитудами плазмонов и экситонов согласно (14). Зависимость от времени амплитуд (20) в плазмон-экситоне 
определяется полюсами (19) функции $D^{-1}(p)$, а функции $M_{n}(p)$ из (14) полюсов не имеют. При этих условиях выражение (20) дает

$$
\begin{aligned}
w_{n}(t) & =2 \operatorname{Re} \sum_{k=1,3} e^{p_{k} t} \Phi_{n}\left(p_{k}\right) \prod_{\substack{k^{\prime}=1 \\
\left(k^{\prime} \neq k\right)}}^{4} \frac{1}{p_{k}-p_{k^{\prime}}} \\
& =\sum_{\nu=\mathrm{I}, \mathrm{II}} e^{-g_{v} t} \operatorname{Re}\left[e^{-i u_{v} t} U_{n}\left(p_{\nu}\right)\right]
\end{aligned}
$$

для плазмонной $(n=1)$ и экситонной $(n=2)$ амплитуд в смешанных модах. Суммирование проводится по парам комплексно сопряженных полюсов из (19), т. е. берется $p_{\mathrm{I}}=p_{1}$ для моды с $v=\mathrm{I}$ и $p_{\mathrm{II}}=p_{3}$ для моды с $v=\mathrm{II}$. В формуле (23)

$$
\left.\begin{array}{c}
U_{n}\left(p_{v}\right)=2 \Phi_{n}\left(p_{v}\right) H_{v} \equiv U_{n v}, \\
\Phi_{1}(p)=\left(p^{2}+p \Gamma_{2}+\omega_{22}^{2}\right) M_{1}(p)-\Delta \omega^{2} M_{2}(p), \\
\Phi_{2}(p)=-\Delta \omega^{2} M_{1}(p)+\left(p^{2}+p \Gamma_{1}+\omega_{11}^{2}\right) M_{2}(p) .
\end{array}\right\}
$$

С учетом выражений (21) и (22) находим

$$
\begin{aligned}
H_{v} & =\left.\frac{1}{p_{v}-p_{v}^{*}} \frac{1}{\left(p_{v}-p_{v^{\prime}}\right)\left(p_{v}-p_{v^{\prime}}^{*}\right)}\right|_{\substack{v^{\prime}=\mathrm{IIII} \\
v^{\prime} \neq v}} \\
& =\frac{i s_{v}}{2 u_{v}} \frac{1}{u_{\mathrm{II}}^{2}-u_{\mathrm{I}}^{2}-2 i u_{v}\left(g_{\mathrm{II}}-g_{\mathrm{I}}\right)} .
\end{aligned}
$$

Здесь $s_{\text {I }}=1, s_{\text {II }}=-1$ и

$$
g_{\text {II }}-g_{\text {I }}=\frac{\Gamma_{2}-\Gamma_{1}}{2} \frac{\omega_{22}^{2}-\omega_{11}^{2}}{\sqrt{\left(\omega_{22}^{2}-\omega_{11}^{2}\right)^{2}+4 \Delta \omega^{4}}} .
$$

Из формулы (27) очевиден следующий результат:

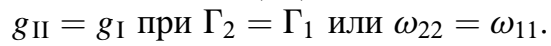

Формула (23) для функций релаксации плазмонов и экситонов, входящих в плазмон-экситонные моды $v=\mathrm{I}$ и II, принимает вид

$$
\begin{aligned}
w_{n}(t) & =\sum_{\nu=\mathrm{I}, \mathrm{II}} w_{n}^{(v)}(t) \\
& =\sum_{\nu=\mathrm{I}, \mathrm{II}}\left|U_{n v}\right| e^{-g_{v} t} \cos \left(u_{v} t-\phi_{n v}\right),
\end{aligned}
$$

где $\phi_{n v}=\operatorname{arctg}\left[\operatorname{Im} U_{n v} / \operatorname{Re} U_{n v}\right]$. Таким образом, слагаемые $w_{n}^{(v)}$ с $v=$ I или II в сумме (28) выражают вклад плазмонов $(n=1)$ и экситонов $(n=2)$ в $v$-ю смешанную моду, которая имеет частоту $u_{v}$ и время релаксации по энергии $1 /\left(2 g_{v}\right)$.

Заметим, что если $\Delta \omega=0$ в уравнениях (13), то (28) переходит в выражения $\tilde{w}(t)$ для невзаимодействующих плазмонов $(n=1)$ и экситонов $(n=2)$. Для них имеем

$$
\widetilde{w}_{n}(t)=e^{-\Gamma_{n} t / 2}\left[\widetilde{w}_{n}^{0} \cos \left(\omega_{n n} t\right)+\left(\widetilde{w}_{n}^{0} \frac{\Gamma_{n}}{2}+\dot{\tilde{w}}_{n}^{0}\right) \frac{\sin \left(\omega_{n n} t\right)}{\omega_{n n}}\right] .
$$

Формула (29) выражает общее решение задачи о затухающих колебаниях осциллятора с частотой $\omega_{n n}$ и параметром затухания $\Gamma_{n}$ при начальных условиях $\widetilde{w}_{n}(0)=\widetilde{w}_{n}^{0}$ и $\dot{\widetilde{w}}_{n}(0)=\dot{\widetilde{w}}_{n}^{0}$.

\section{5. Обсуждение результатов}

Проанализируем численно решение задачи о релаксации плазмонной и экситонной компонент, образующих смешанные плазмон-экситонные моды с $v=$ I и II. При вычислении зависимостей (28) от времени используем комплексные частоты мод $u_{v}-i g_{v}$ из (21) и (22) и величины $U_{n v}$ из (24)-(27), которые учитывают плазмонэкситонное взаимодействие с помощью константы (15). Параметры 2D-плазмон-экситонов принимаем близкими к тем, что использовались в [10] для квази 2D-возбуждений. Для $2 \mathrm{D}$-плазмонов в слое наномеровой толщины $L$ считаем, что $n_{2 \mathrm{D}} \approx n_{3 \mathrm{D}} L$, где $n_{3 \mathrm{D}}-$ плотность $3 \mathrm{D}$ электронного газа металла.

На рис. 2, $a$ и 2, $b$ представлены вычисленные по формулам (21) и (22) законы дисперсии $u_{v}(\kappa)$ и параметры затухания $g_{v}(\kappa)$ плазмон-экситонов с $v=$ I и II. Интерес представляет поведение этих зависимостей для мод, имеющих волновые числа вблизи значения $\kappa$, которое соответствует резонансу $\omega_{11}(\kappa)=\omega_{22}(\kappa)$ между плазмонами и экситонами. Вблизи резонанса в отсутствие затухания рис. 2, $a$ показывает эффект антипересечения дисперсионных ветвей $u_{\mathrm{I}}(\kappa)$ и $u_{\mathrm{II}}(\kappa)$ для нормальных мод плазмон-экситонов. Из рис. $2, b$ видно, что величины параметров затухания $g_{v}$ плазмон-экситонов в области антипересечения перераспределяются между плазмоноподобной и экситоноподобной компонентами, причем при резонансе выполняется условие $g_{\text {I }}=g_{\text {II }}$ в согласии с формулой (27).

Вдали от резонанса $\left(\Gamma_{n} \ll\left|\omega_{22}-\omega_{11}\right|\right)$ временны́е зависимости процессов релаксации невзаимодействующих плазмонов $(n=1)$ или экситонов $(n=2)$ приближенно описываются элементарной формулой (29). В случае экситонов большого радиуса параметры затухания обычно удовлетворяют условию $\Gamma_{2} \ll \Gamma_{1}$, т.е. экситоны релаксируют за время $1 / \Gamma_{2}$ существенно медленнее, чем плазмоны за время $1 / \Gamma_{1}$.

Вблизи резонанса $\omega_{11}=\omega_{22}$ возбуждения и процессы их релаксации модифицируются из-за плазмон-экситонного взаимодействия. Согласно $(24)-(27)$ величины $U_{n v}$ зависят от характера начального возбуждения смешанных мод с $v=$ I или II, каждая из которых является линейной комбинацией плазмона и экситона. Поэтому следует иметь в виду, что возбуждение любой из парциальных компонент $w_{n}^{(v)}$ с $n=1$ и 2 на самом деле означает возбуждение $v$-й смешанной моды как целого. Различие между компонентами $w_{n}^{(v)}(t)$ определяется величинами $U_{n v}$, которые отражают особенности плазмон-экситонного энергообмена в смешанной моде. Формула (28) показывает, что амплитуды $w_{n}^{(v)}(t)$ плазмона $(n=1)$ и экситона $(n=2)$ в смешанной моде с 

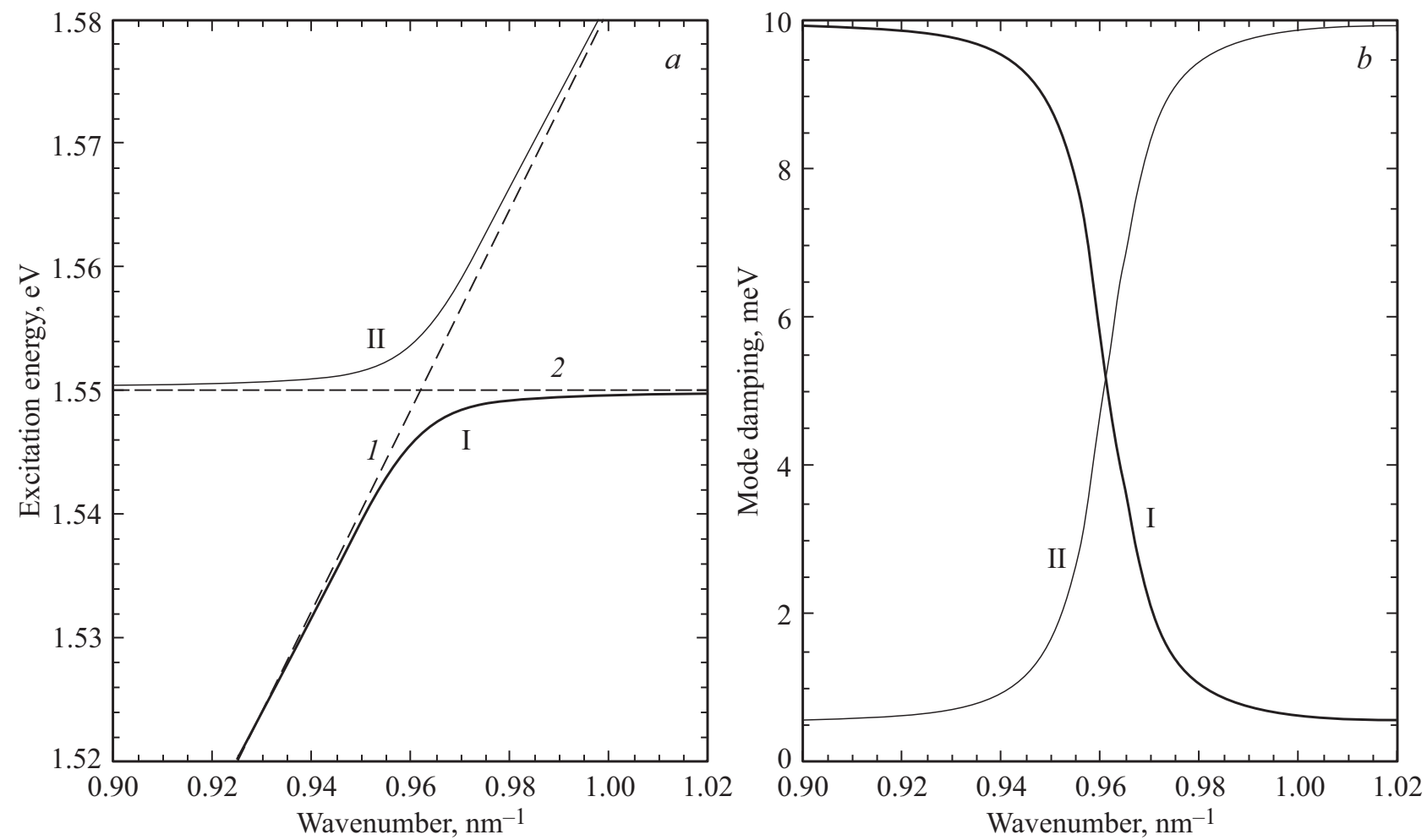

Рис. 2. (a) Законы дисперсии $\hbar u_{\mathrm{I}}$ (I) и $\hbar u_{\mathrm{II}}(\mathrm{II})$ нормальных волн $2 \mathrm{D}-$ плазмон-экситонов с волновыми числами $\kappa$ при $\Gamma_{1}=\Gamma_{2}=0$. Пунктиром показаны энергии парциальных $2 \mathrm{D}-$ плазмонов $\hbar \omega_{2 \mathrm{D}}(1)$ и экситонов $\hbar \omega_{0}(2)$. (b) Параметры затухания $\hbar g_{\mathrm{I}}(\mathrm{I})$ и $\hbar g_{\text {II }}(\mathrm{II})$

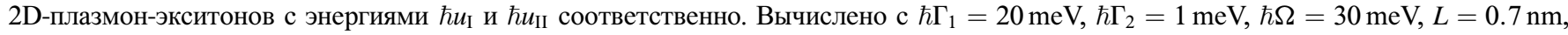
$l=h=1 \mathrm{~nm}$ и теми же другими параметрами, что на рис. 1.

номером $v$ и частотой $u_{v}$ экспоненциально затухают за время $1 / g_{v}$.

Для примера рассмотрим релаксацию мод поляризации плазмон-экситонов, предполагая, что при $t=0$ возбуждена волна экситонной поляризации из (9) с вектором $\kappa$ и амплитудой $w_{2}^{0} \neq 0$. Полагаем также, что $w_{1}^{0}=\dot{w}_{1}^{0}=\dot{w}_{2}^{0}=0$ при $t=0$, тогда $M_{1}(p)=0$, $M_{2}(p)=\left(p+\Gamma_{2}\right) w_{2}^{0}$ в формулах (14) и (25). Отметим, что по возбуждению 2D-экситонов имеются эксперименты, например [23], где используется ближнеполевое возбуждение с участием плазмонных поляритонов.

Зависимости от $\kappa$ величин $\left|U_{n v}\right|$, вычисленных по формулам (24)-(27) и нормированных на $w_{2}^{0}$, показаны на рис. 3 для смешанных мод $v=$ I и II, спектры которых представлены на рис. 2. Из рис. 3 видно, что если при $t=0$ возбужден экситон $\left(w_{2}^{0} \neq 0\right)$, то в модах с $\kappa$ вдали от резонанса $w_{11}(\kappa)=\omega_{22}(\kappa)$ существенны экситонные компоненты $\left|U_{2 v}\right|$, а плазмонные $\left|U_{1 v}\right|$ подавлены. В непосредственной близости к резонансу величины $\left|U_{2 v}\right|$ перераспределяются между модами $v=\mathrm{I}$ и II, а величины $\left|U_{1 v}\right|$ для плазмонных составляющих этих мод значительно возрастают. При заданном $n$ функции $\left|U_{n v}\right|$ от $\kappa$ для мод с разными $v$ имеют характерную симметрию относительно значения $\kappa$, соответствующего плазмон-экситонному резонансу $\omega_{11}(\kappa)=\omega_{22}(\kappa)$. Возрастание плазмонных составляющих $\left|U_{1 v}\right|$ в области резонанса (антипересечения) означает ускорение релак- сации плазмон-экситонов через относительно быстро затухающую плазмонную компоненту.

Проявление этих особенностей во временно́й зависимости релаксации плазмон-экситонов иллюстрирует рис. 4. На нем изображены затухающие при $t>0$

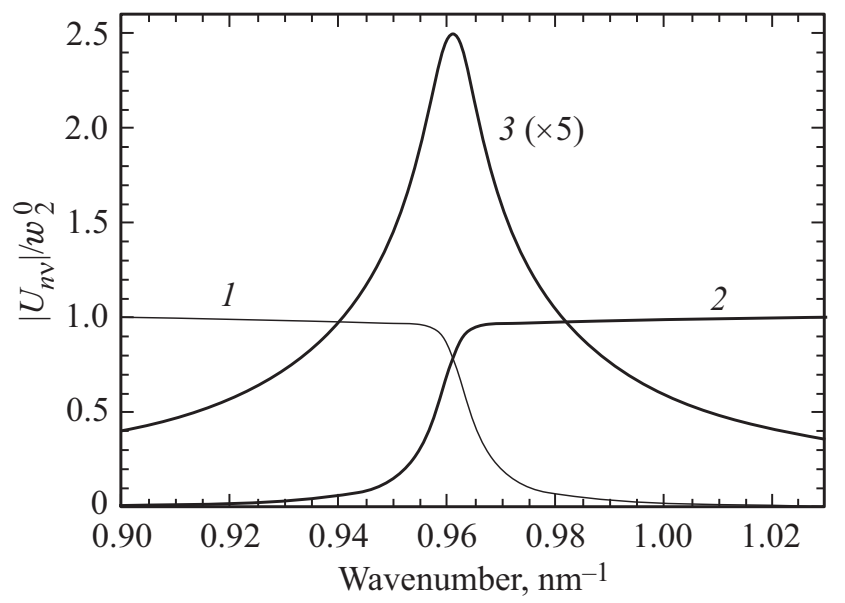

Рис. 3. Зависимости от волнового числа амплитуд $\left|U_{n v}\right| / w_{2}^{0}$ плазмонов $(n=1)$ и экситонов $(n=2)$ в плазмон-экситонных модах с $v=$ I и II. Функции $\left|U_{2, \mathrm{II}}\right|(1),\left|U_{2, \mathrm{I}}\right|(2),\left|U_{1, \mathrm{I}}\right|$ и $\left|U_{1, \mathrm{II}}\right|$ (3, умножено на 5) нормированы на амплитуду экситона $w_{2}^{0}$, возбужденного при $t=0$. Вычислено с теми же параметрами, что на рис. 2. 


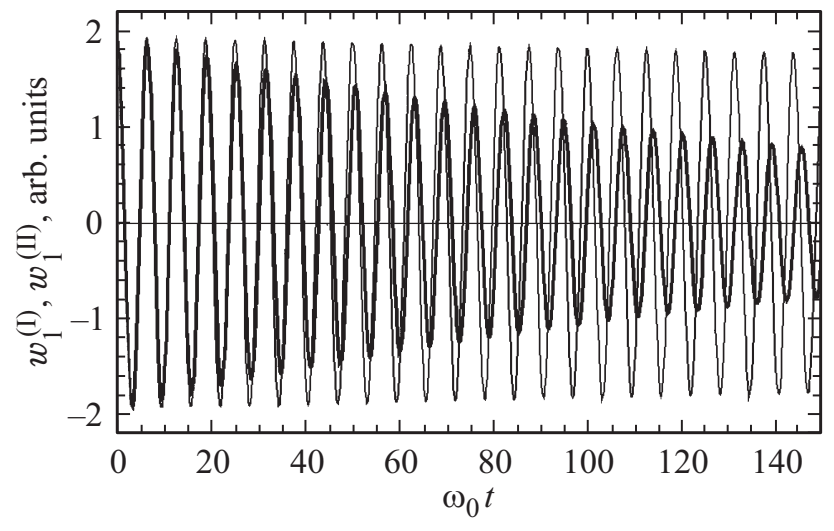

Рис. 4. Зависимость от времени $\omega_{0} t$ затухающих амплитуд $w_{1}^{(\mathrm{I})}$ (жирная линия) и $w_{1}^{\text {(II) }}$ (тонкая линия) плазмонной поляризации в плазмон-экситонных модах с $v=$ I и II соответственно. Вычислено с теми же параметрами, что на рис. 2, в предположении, что при $t=0$ был возбужден экситон с $w_{2}^{0} \neq 0$ и $\kappa=0.95 \mathrm{~nm}^{-1}$.

плазмонные компоненты $w_{1}^{(v)} \sim \exp \left(-g_{v} t\right)$ в смешанных модах $v=$ I, II при условии, что первоначально был возбужден экситон $\left(w_{2}^{0} \neq 0\right)$ с $\kappa=0.95 \mathrm{~nm}^{-1}$. Видно, что амплитуда $w_{1}^{(\mathrm{I})} \sim \exp \left(-g_{\mathrm{I}} t\right)$ моды с $v=\mathrm{I}$, которая является плазмоноподобной согласно рис. 2, $a$, затухает значительно быстрее, чем амплитуда $w_{1}^{(\mathrm{II})} \sim \exp \left(-g_{\mathrm{II}} t\right)$ экситонооподобной моды с $v=$ II. Этот вывод находится в согласии с рис. $2, b$, из которого следует, что $g_{\mathrm{I}} \gg g_{\text {II }}$ при $\kappa=0.95 \mathrm{~nm}^{-1}$.

\section{6. Заключение}

Выше представлена теория двумерных плазмонэкситонов и процессов их релаксации в зависимости от времени. Сформулированы уравнения, описывающие возбуждение и затухание плазмон-экситонных мод в классической модели связанных осцилляторов, роль которых играют волны поляризации двумерных плазмонов и экситонов. Для этих поляризационных возбуждений найдена константа взаимодействия (кулоновской связи), которая обеспечивает образование смешанных плазмонэкситонных мод. Поведение частот нормальных мод плазмон-экситонов при прохождении резонанса между плазмоном и экситоном имеет характерные признаки антипересечения частот. При учете затухания парциальных плазмонов и экситонов предполагалось, что вдали от их резонанса спектр состоит из узкого экситонного и широкого плазмонного уровней энергии. Показано, что при резонансе они трансформируются в два плазмонэкситонных уровня одинаковой ширины, симметричных относительно частоты резонанса.

Представленная теория плазмон-экситонов в модели связанных гармонических осцилляторов делает прозрачной их аналогию с возбуждениями других систем линейной теории колебаний (механические осцилляторы, элекрические контуры и др. [24]). В частности, это важно для обобщения теории низкоразмерных плазмонов, экситонов и плазмон-экситонов на периодические и неупорядоченные структуры металл-полупроводник.

\section{Конфликт интересов}

У автора отсутствует конфликт интересов.

\section{Список литературы}

[1] A.A. Toropov, T.V. Shubina. Plasmonic Effects in MetalSemiconductor Nanostructures. Oxford Univ. Press (2015). $371 \mathrm{p}$.

[2] M. Achermann. J. Phys. Chem. Lett. 1, 2837 (2010).

[3] B.G. DeLacy, O.D. Miller, C.W. Hsu, Z. Zander, S. Lacey, R. Yagloski, A.W. Fountain, E. Valdes, E. Anquillare, M. Soljacic, S.G. Johnson, J.D. Joannopoulos. Nano Lett. 15, 2588 (2015).

[4] A.O. Govorov, G.W. Bryant, W. Zhang, T. Skeini, J. Lee, N.A. Kotov, J.V. Slocik, R.R. Naik. Nano Lett. 6, 5, 984 (2006).

[5] V.A. Kosobukin. Solid State Commun. 228, 43 (2016).

[6] E.L. Ivchenko. Optical spectroscopy of semiconductor nanostructures. Alpha Science International Ltd. (2005). 427 p.

[7] Поверхностные поляритоны. Электромагнитные волны на поверхностях и границах раздела сред. / Под ред. В.М. Аграновича и Д.Л. Миллса. Наука, М. (1985). 525 с.

[8] В.М. Агранович, В.Л. Гинзбург. Кристаллооптика с учетом пространственной дисперсии и теория экситонов. Наука, M. (1979). $432 \mathrm{c.}$

[9] U. Kreibig, M. Vollmer. Optical properties of metal clusters. Springer (1995). $532 \mathrm{p}$.

[10] В.А. Кособукин. ФТТ 63, 4, 527 (2021).

[11] J. Bellessa, C. Bonnand, J.C. Plenet, J. Mugnier. Phys. Rev. Lett. 93, 3, 036404 (2004).

[12] S. Balci. Opt. Lett. 38, 21, 4498 (2013).

[13] B.J. Lawrie, K.-W. Kim, D.P. Norton, R.F. Haglund Jr. Nano Lett. 12, 6152 (2012).

[14] М.Г. Кучеренко, Т.М. Чмерева. Оптика и спектроскопия 125, 2, 165 (2018).

[15] В.М. Агранович, М.Д. Галанин. Перенос энергии электронного возбуждения в конденсированных средах. Наука, М. (1978). 383 c.

[16] Т. Андо, Ф. Фаулер, Ф. Стерн. Электронные свойства двумерных систем. Мир, М. (1985). 415 с.

[17] В.А. Кособукин. ФТТ 28, 11, 3516 (1986).

[18] W.G. Teich, G. Mahler. Phys. Status Solidi B 138, 2, 607 (1986).

[19] В.А. Кособукин, А.Н. Поддубный. ФТТ 49, 10, 1883 (2007).

[20] Е.Л. Ивченко. ФТТ 33, 8, 2388 (1991).

[21] E.S. Khramtsov, P.A. Belov, P.S. Grigoryev, I.V. Ignatiev, S.Yu. Verbin, Yu.P. Efimov, S.A. Eliseev, V.A. Lovtcius, V.V. Petrov, S.L. Yakovlev. J. Appl. Phys. 119, 18, 184301 (2016).

[22] М.А. Лаврентьев, Б.В. Шабат. Методы теории функций комплексного переменного. Наука, М. (1987). 688 с.

[23] Y. Zhou, G. Scuri, D.S. Wild, A.A. High, A. Dibos, L.A. Jauregui, C. Shu, K. De Greve, K. Pistunova, A.Y. Joe, T. Taniguchi, K. Watanabe, P. Kim, M.D. Lukin, H. Park. Nat. Nanotechnol. 12, 856 (2017).

[24] С.П. Стрелков. Введение в теорию колебаний. Наука, М. (1964). $437 \mathrm{c}$.

Редактор Ю.Э. Китаев 